\title{
IL17a and IL21 combined with surgical status predict the outcome of ovarian cancer patients
}

\author{
Yu-Li Chen ${ }^{1,2,3}$, Cheng-Yang Chou ${ }^{4}$, Ming-Cheng Chang ${ }^{3}$, Han-Wei Lin ${ }^{5}$, \\ Ching-Ting Huang ${ }^{5}$, Shu-Feng Hsieh ${ }^{3}$, Chi-An $\mathrm{Chen}^{3}$ and Wen-Fang Cheng ${ }^{3,5,6}$ \\ ${ }^{1}$ College of Medicine, Graduate Institute of Anatomy and Cell Biology, National Taiwan University, No. 1, Section 1, \\ Jen-Ai Road, Taipei, Taiwan \\ ${ }^{2}$ Department of Obstetrics and Gynecology, Gynecologic Cancer Center, Cathay General Hospital, Taipei, Taiwan \\ ${ }^{3}$ Department of Obstetrics and Gynecology, College of Medicine, National Taiwan University, No. 1, Section 1, \\ Jen-Ai Road, Taipei, Taiwan \\ ${ }^{4}$ Department of Obstetrics and Gynecology, College of Medicine and the Hospital, National Cheng Kung University, \\ Tainan, Taiwan \\ ${ }^{5}$ College of Medicine, Graduate Institute of Oncology, and ${ }^{6}$ College of Medicine, Graduate Institute of Clinical \\ Medicine, National Taiwan University, No. 1, Section 1, Jen-Ai Road, Taipei, Taiwan
}

\author{
Correspondence \\ should be addressed \\ to C-A Chen or W-F Cheng \\ Emails \\ chianchen@ntu.edu.tw or \\ wenfangcheng@yahoo.com
}

\begin{abstract}
Aside from tumor cells, ovarian cancer-related ascites contains the immune components. The aim of this study was to evaluate whether a combination of clinical and immunological parameters can predict survival in patients with ovarian cancer. Ascites specimens and medical records from 144 ovarian cancer patients at our hospital were used as the derivation group to select target clinical and immunological factors to generate a risk-scoring system to predict patient survival. Eighty-two cases from another hospital were used as the validation group to evaluate this system. The surgical status and expression levels of interleukin 17a (IL17a) and IL21 in ascites were selected for the risk-scoring system in the derivation group. The areas under the receiver operating characteristic (AUROC) curves of the overall score for disease-free survival (DFS) of the ovarian cancer patients were 0.84 in the derivation group, 0.85 in the validation group, and 0.84 for all the patients. The AUROC curves of the overall score for overall survival (OS) of cases were 0.78 in the derivation group, 0.76 in the validation group, and 0.76 for all the studied patients. Good correlations between overall risk score and survival of the ovarian cancer patients were demonstrated by sub-grouping all participants into four groups ( $P$ for trend $<0.001$ for DFS and OS). Therefore, acombination of clinical and immunological parameters can provide a practical scoring system to predict the survival of patients with ovarian carcinoma. IL17a and IL21 can potentially be used as prognostic and therapeutic biomarkers.
\end{abstract}

Endocrine-Related Cancer (2015) 22, 703-711

\section{Introduction}

Ovarian carcinoma is the most lethal gynecologic malignancy (Jemal et al. 2011). The standard treatment for this disease is debulking surgery followed by platinum-based chemotherapy; however, the overall survival (OS) rate is around 35\% (Cannistra 2004, Kim et al. 2012). The high mortality rate is due to vague symptoms, diagnosis at a late stage and a lack of accurate biomarkers (Suh et al. 2010, Wang et al. 2015). An effective diagnosis may be made via

Published by Bioscientifica Ltd 
a combination of robust biomarkers specific for ovarian cancer and current clinical methods. Therefore, further research to identify accurate ovarian cancer biomarkers is needed to allow for early detection and diagnosis and subsequently better care. Furthermore, these biomarkers would be prognostic factors to predict the disease course and the response to treatment (Bast et al. 2009, Suh et al. 2010).

Cancer arises in the tumor microenvironment that is both a cause and a consequence of tumorigenesis. Tumor and host cells co-evolve dynamically through indirect and direct cellular interactions (Casey et al. 2015). Many of the hallmarks of cancer are related to the microenvironment, including the ability to induce proliferation and angiogenesis, and avoid apoptosis, hypoxia, and immune detection (Hanahan \& Weinberg 2011). Ovarian cancer is characterized to spread primarily by tumor cell implantation in the peritoneal cavity. In addition to solid tumors, ascites is also frequently noted in patients with ovarian cancer. In these fluids, several kinds of tumor-associated cells including immunocytes and also regulatory elements such as cytokines and chemokines are found in ascites. Therefore, ascites can be considered to be part of the tumor microenvironment and ideal to adequately reflect the relationship between host immunity and tumor cells in the cancer microenvironment (Yigit et al. 2011, Fridman et al. 2012).

Ovarian carcinoma has been demonstrated to be immunogenic, however the underlying interaction between host immunity and tumor-associated cells is not clear (Zhang et al. 2003, Curiel et al. 2004, Tomsová et al. 2008). The presence of tumor infiltrating lymphocytes has been positively correlated with survival in ovarian cancer patients (Zhang et al. 2003, Raspollini et al. 2005). However, ovarian cancer can create an immunosuppressive microenvironment to escape immune elimination (Latha et al. 2014). One of the escape mechanisms is a shift in the immune response from helper $\mathrm{T} 1$ (Th1) toward Th2, a process mediated by cytokines (Yigit et al. 2011). A lower level of the pro-inflammatory Th17-related cytokine, interleukin 17 (IL17), has also been reported in more advanced ovarian cancer-associated ascites (Hirahara et al. 2001, Kryczek et al. 2009). In addition to modulating the status of host immunity, alterations of Th1-, Th2-, and Th17-related cytokines in ascites can be considered to be immune biomarkers to evaluate disease severity and prognosis (Kryczek et al. 2009, Yigit et al. 2011, Cândido et al. 2013, Chen et al. 2013).

Few studies have reported using a combination of clinical and immunological parameters to predict survival in ovarian cancer patients. The aim of this study was to evaluate whether a combination of clinical parameters and cytokine profiles in ascites can predict survival in patients with ovarian cancer. Therefore, Th1 (interferon gamma (IFN $\gamma$ ) and tumor necrosis factor alpha (TNF $\alpha)$ )-, Th2 (IL4, IL6, and IL10)-, and Th17 (IL17a, IL17f, IL21, IL22, and IL23)-related cytokines were selected for analysis in this study (Dong 2008, Oreja-Guevara et al. 2012). Ascites specimens and medical records from 144 ovarian cancer patients in our hospital were used as the derivation group to select target clinical and immunological factors to generate a risk-scoring system to predict patient survival. Eighty-two cases from another hospital were used as the validation group to evaluate this risk-scoring system. The clinical application of this risk-scoring system was also discussed.

\section{Materials and methods}

\section{Patients and specimens}

From July 2003 to September 2012, 226 women diagnosed with ovarian carcinoma who received debulking or staging surgery were enrolled. One hundred and forty-four of the 226 patients were from National Taiwan University Hospital (NTUH) in northern Taiwan, and the remaining 82 cases were from National Cheng Kung University Hospital (NCKUH) in southern Taiwan. All of the patients who received neoadjuvant chemotherapy before surgery were excluded. The Institutional Review Boards of both hospitals approved the study protocol. Ascites specimens were collected during surgery and centrifuged at $760 \boldsymbol{g}$ for $5 \mathrm{~min}$ into cellular elements and supernatant. The supernatant was kept at $-20^{\circ} \mathrm{C}$ until analysis (Chen et al. 2012a,b).

The medical records of the patients were prospectively reviewed until December 2013 to obtain clinical parameters including age, operative findings, pathological findings, disease relapse, and prognosis. The characteristics of disease were defined according to the system of International Federation of Gynecology and Obstetrics (FIGO) (1971). Stage I and II diseases were considered to be early stage, and stages III and IV as advanced stage. Except for the women with stage IA and grade I tumors, all patients received three to six courses of adjuvant platinum-based chemotherapy. The maximum diameters of postoperative residual tumors were also recorded. When the maximum diameter of a residual tumor was $\leq 1 \mathrm{~cm}$, the surgical status was defined as being optimal debulking surgery. Otherwise, the surgical status was defined as sub-optimal.

Published by Bioscientifica Ltd. 
After completion of the primary treatment, history taking, pelvic/rectal examination, and regional lymph nodal palpation would be arranged every 3 months for 3 years, and every 6 months thereafter. Serum levels of carcinoembryonic antigen and carcinoma antigen 125 were measured at each visit, and smears of the vaginal cuff were performed annually. Magnetic resonance imaging or computerized tomography was arranged for suspected disease relapse. Recurrence was considered when tumor marker levels were greater than or equal to twofold the upper limit of normal in two consecutive tests with 2-week intervals, findings of imaging studies and aspiration cytology were abnormal, or when there was histological confirmation from a tissue biopsy. The period of time from completion of the primary treatment until the diagnosis of disease recurrence was defined as disease-free survival (DFS). The time from the diagnosis of disease until the date of death or last visit was defined as OS (Chen et al. $2012 a, b)$.

\section{Cytokine measurements (cytokine bead array)}

In this study, ten cytokines including IFN $\gamma, \mathrm{TNF} \alpha$, IL4, IL6, IL10, IL17a, IL17f, IL21, IL22, and IL23 were evaluated. Levels of these cytokines were measured using a customized Procarta cytokine profiling kit (Affimatrix, Santa Clara, CA, USA) as in our previous study (Chen et al. 2013). In the assays, the threshold of detection for the various cytokines ranged from 0.10 to $0.92 \mathrm{pg} / \mathrm{ml}$ respectively. Intra- and inter-assay coefficients of variation for these cytokines were $<10.0 \%$. The details were shown in Supplementary Table 1 , see section on supplementary data given at the end of this article.

\section{Development of the risk-scoring system}

The studied population was divided into two groups, participants from NTUH as the NTUH set (derivation group) and those from NCKUH as the NCKUH set (validation group). There were two steps for the development of the risk-scoring system that was modified from the methodology of Chan et al. (2014).

First, a univariate Cox proportional hazard model was used to select the significant risk predictors for the OS of the derivation group. The expression levels of all cytokines were divided into high and low groups by median levels in order to facilitate statistical analysis. The median levels for IFN $\gamma$, TNF $\alpha$, IL4, IL6, IL10, IL17a, IL17f, IL21, IL22, and IL23 were 4.5, 2.9, 2.5, 942.5, 5.8, $16.9,21.3,135.2,18.0$, and $16.0 \mathrm{pg} / \mathrm{ml}$ respectively.
Tests for proportional hazards assumption of the Cox regression model were performed. All of the risk predictors were time-independent (Supplementary Table 2, see section on supplementary data given at the end of this article). Moreover, the effects of product terms (Supplementary Table 3) and the correlation coefficients (Supplementary Table 4) between clinical parameters (FIGO stage, histology, tumor grade, and optimal surgery) and dichotomized immunological factors were evaluated.

Second, multivariate Cox regression analysis was performed including the significant risk predictors selected from the univariate analysis. The regression coefficients of significant risk factors in the multivariate analysis were divided by the smallest regression coefficient (for IL21 in this study) before being rounded to an integer value to generate the risk scores.

The sensitivity and specificity of the predictive scores for disease relapse and disease-related death of the women with ovarian cancer were estimated using receiver operating characteristic (ROC) curves. We calculated the areas under the ROC curves (AUROC) of the derivation group, validation group, and all patients. To evaluate the validity of the scoring system in the prediction of clinical outcomes of the ovarian cancer patients, all of the cases were categorized into four groups based on the predictive scores. The survival functions of different groups were compared by Cox proportional hazard regression.

\section{Statistical analysis}

All statistical analyses were performed using SPSS for Windows version 15.0 (SPSS, Inc.). The clinicopathological characteristics between two sets were compared using the $\chi^{2}$ test for dichotomized variables and the Mann-Whitney $U$ test for continuous variables. The expression levels of the cytokines between two sets or among various histological subtypes were analyzed by the Mann-Whitney $U$ test or Kruskal-Wallis test. Statistical significance was considered as $P<0.05$.

\section{Results}

\section{Characteristics of the studied population}

In total, 226 women with ovarian cancer were enrolled as the study population, of whom 144 were in the NTUH set and 82 in the NCKUH set. The clinicopathological characteristics of these cases are shown in Table 1 . The mean follow-up duration was 29.3 months in the NTUH

Published by Bioscientifica Ltd. 
Table 1 Clinicopathological characteristics of the studied ovarian carcinoma patients

\begin{tabular}{l} 
Characteristic \\
\hline Follow-up period*,a (mean \pm s.D., months) \\
Patients \\
Age ${ }^{*, a}$ (mean \pm s.D., years) \\
Disease status (cases (\%)) \\
FIGO stage ${ }^{*, b}$ \\
Early \\
Advanced \\
Histology ${ }^{*, \mathrm{~b}}$ \\
Serous \\
Mucinous \\
Clear cell \\
Endometrioid \\
Undifferentiated \\
Tumor grade*,b,c \\
1 \\
2 \\
3 \\
Debulking surgery ${ }^{\dagger, \mathrm{b}}$ \\
Optimal \\
Suboptimal \\
Outcome (cases (\%)) \\
Disease recurrence \\
Yes \\
No \\
Disease-related death*,b \\
Yes \\
No
\end{tabular}

\begin{tabular}{c}
\hline NTUH set $(n=144)$ \\
\hline $29.3 \pm 22.0$ \\
$54.5 \pm 11.6$ \\
\\
$45(31.2 \%)$ \\
$99(68.8 \%)$ \\
$65(45.1 \%)$ \\
$5(3.5 \%)$ \\
$35(24.3 \%)$ \\
$21(14.6 \%)$ \\
$18(12.5 \%)$ \\
$20(14.2 \%)$ \\
$13(9.2 \%)$ \\
$108(76.6 \%)$ \\
$86(59.7 \%)$ \\
$58(40.3 \%)$ \\
$72(50 \%)$ \\
$72(50 \%)$ \\
$38(26.4 \%)$ \\
$106(73.6 \%)$ \\
\end{tabular}

\begin{tabular}{c} 
NCKUH set $(n=82)$ \\
\hline $33.7 \pm 23.0$ \\
$55.2 \pm 12.5$ \\
$24(29.3 \%)$ \\
$58(70.7 \%)$ \\
$42(51.2 \%)$ \\
$8(9.8 \%)$ \\
$15(18.3 \%)$ \\
$12(14.6 \%)$ \\
$5(6.1 \%)$ \\
$4(5.3 \%)$ \\
$8(10.7 \%)$ \\
$63(84 \%)$ \\
$63(76.8 \%)$ \\
$19(23.2 \%)$ \\
\\
$46(56.1 \%)$ \\
$36(43.9 \%)$ \\
$29(35.4 \%)$ \\
$53(64.6 \%)$ \\
\end{tabular}

Studied population $(n=226)$

$30.9 \pm 22.4$

$54.8 \pm 12.0$

$69(30.5 \%)$

$157(69.5 \%)$

$107(47.3 \%)$

$13(5.8 \%)$

$50(22.1 \%)$

$33(14.6 \%)$

$23(10.2 \%)$

$24(11.1 \%)$

$21(9.7 \%)$

$171(79.2 \%)$

$149(65.9 \%)$

$77(34.1 \%)$

$P>0.05$ and ${ }^{\dagger} P=0.01$. F

Kung University Hospital.

By Mann-Whitney $U$ test.

${ }^{b}$ By $\chi^{2}$ test.

'Unavailable data for three patients in NTUH set and seven patients in NCKUH.

set and 33.7 months in the NCKUH set. The mean age at diagnosis of ovarian cancer was 54.5 years in the NTUH set and 55.2 years in the NCKUH set. There were no significant differences in the distribution of disease status, including FIGO stage, histology, and tumor grade or the percentages of disease recurrence and disease-related death, between the two sets. More patients (76.8\%) in the NCKUH set received optimal surgery than in the NTUH set $(59.7 \% ; P=0.01)$.

The median levels of the ten cytokines from the 226 samples of ovarian carcinoma-related ascites are presented in Table 2. The expression levels of all cytokines in ascites were different between the two sets except for IL6. However, the higher or lower levels of cytokine expression were not particularly confined to one medical institute. As shown in Table 3, the IFN $\gamma$, IL10, IL17a, and IL23 levels of patients with various histological subtypes were different. The patients with serous ovarian carcinoma tended to have higher expression levels of IFN $\gamma$ and IL10 and lower expression levels of IL17a in their ascites.

\section{Development of the risk-scoring system with a combination of surgical status and IL17a and IL21 levels}

The results of univariate and multivariate Cox regression analyses to evaluate the risk predictors for the OS of cases in the NTUH set (derivation group) are shown in Tables 4 and 5. In univariate analysis, advanced disease, grade 3 tumors, no optimal (sub-optimal) surgery and dichotomized immunologic parameters of IFN $\gamma$, IL17a, and IL21 expressed in the cancer-associated ascites were significantly associated with the OS of the 144 women (Table 4). In multivariate analysis, sub-optimal cytoreductive surgery (hazard ratio (HR): 2.3 (95\% CI 1.01-5.11), $P=0.048$ ) had a significant impact on the OS of the patients. The other two significant predictors for OS were lower expression levels of IL17a (HR: 3.0 (95\% CI 1.10-8.03), $P=0.032)$ and higher expression levels of IL21 (HR: 2.2 (95\% CI 1.02-4.59), $P=0.045$ ). Therefore, surgical status and the expression levels of IL17a and IL21 in ascites were selected to develop the risk-scoring system in the derivation group (Table 5).

Published by Bioscientifica Ltd 
Table 2 Expression levels of various cytokines in ascites of the ovarian cancer patients

\begin{tabular}{l}
$\begin{array}{l}\text { Cytokines } \\
\text { (median (range), pg/ml) }\end{array}$ \\
\hline IFN $\gamma$ level \\
TNF $\alpha$ level \\
IL4 level \\
IL6 level \\
IL10 level \\
IL17a level \\
IL17f level \\
IL21 level \\
IL22 level \\
IL23 level
\end{tabular}

\begin{tabular}{c}
\hline NTUH set $(n=144)$ \\
\hline $4.5(0.6-339.0)$ \\
$2.9(0.6-77.1)$ \\
$2.5(0.3-73.3)$ \\
$942.5(0.5-15600.0)$ \\
$5.8(0.6-83.3)$ \\
$16.9(1.0-43.2)$ \\
$21.3(13.0-1710.0)$ \\
$135.2(22.0-1206.0)$ \\
$18.0(1.2-130.0)$ \\
$16.0(8.0-770.0)$ \\
\hline
\end{tabular}

\begin{tabular}{c}
\hline NCKUH set $(n=82)$ \\
\hline $26.0(0.2-403.2)$ \\
$5.1(1.8-1600.0)$ \\
$8.6(5.9-720.0)$ \\
$595.0(22.8-15800.0)$ \\
$5.0(3.5-129.0)$ \\
$9.7(2.2-37.2)$ \\
$18.8(16.4-120.0)$ \\
$96.8(11.4-963.0)$ \\
$8.9(3.8-29.4)$ \\
$18.0(10.0-56.0)$ \\
\hline
\end{tabular}

$\boldsymbol{P}$ value $^{\mathrm{a}}$
$<0.001$
$<0.001$
$<0.001$
0.81
0.049
0.04
$<0.001$
0.03
$<0.001$
0.01

\begin{tabular}{c}
$\begin{array}{c}\text { Studied population } \\
(n=226)\end{array}$ \\
\hline $10.8(0.2-403.2)$ \\
$4.0(0.6-1600.0)$ \\
$6.9(0.3-720.0)$ \\
$916.6(0.5-15800.0)$ \\
$5.4(0.6-129.0)$ \\
$15.3(1.0-43.2)$ \\
$20(13.0-1710.0)$ \\
$121.5(11.4-1206.0)$ \\
$13.1(1.2-130.0)$ \\
$17.0(8.0-770.0)$ \\
\end{tabular}

NTUH, National Taiwan University Hospital; NCKUH, National Cheng Kung University Hospital; IFN $\gamma$, interferon gamma; TNF $\alpha$, tumor necrosis factor alpha; $\mathrm{IL}$, interleukin.

aBy Mann-Whitney $U$ test.

Risk scores were calculated as the $\beta$ coefficient of optimal surgery (or IL17a level) divided by the $\beta$ coefficient of IL21 level, and then rounded to an integer value (Table 5). Therefore, sub-optimal surgical status was scored as 1 and optimal status as 0. An IL17a level $<17 \mathrm{pg} / \mathrm{ml}$ in the ascites was scored as 1 and an IL17a level $\geq 17 \mathrm{pg} / \mathrm{ml}$ as 0 . An IL21 level $\geq 135 \mathrm{pg} / \mathrm{ml}$ in the ascites was scored as 1 , and an IL21 level $<135 \mathrm{pg} / \mathrm{ml}$ as 0 . The overall score for each ovarian cancer patient ranged from 0 to 3 .

\section{Validation of the risk-scoring system}

The ROC curves of the overall score for disease recurrence in the derivation group (NTUH set; AUROC, 0.84 ; 95\% CI 0.78-0.91), validation group (NCKUH set; AUROC, 0.85; 95\% CI 0.76-0.94), and all patients (AUROC, 0.84; 95\% CI
0.79-0.89) are shown in Fig. 1A. The ROC curves of the overall score for disease-related death in the derivation group (NTUH set; AUROC, 0.78; 95\% CI 0.69-0.86), validation group (NCKUH set; AUROC, 0.76; 95\% CI $0.66-0.86$ ), and all studied patients (AUROC, 0.76; 95\% CI 0.69-0.82) are shown in Fig. 1B.

\section{Clinical application of the risk-scoring system}

According to the overall scores of the women with ovarian cancer, the 226 patients were divided into four groups. As demonstrated in Fig. 2A, the median DFS was 32 months for the women with an overall score of 0 , 19 months for an overall score of 1, 9 months for an overall score of 2 and 4 months for an overall score of 3 (HR: 3.1 (95\% CI 2.50-3.93), $P$ for trend $\left.\left(P_{\text {trend }}\right)<0.001\right)$. In addition, the median OS was 32 months for the

Table 3 Expression levels of various cytokines in ascites of the ovarian cancer patients with various histological subtypes in all studied population

\begin{tabular}{|c|c|c|c|c|c|c|}
\hline \multirow{2}{*}{$\begin{array}{l}\text { Cytokines } \\
\text { (median (range), } \\
\mathrm{pg} / \mathrm{ml} \text { ) }\end{array}$} & \multicolumn{5}{|c|}{ Histological subtype } & \multirow[b]{2}{*}{$\boldsymbol{P}_{\text {value }}{ }^{\mathrm{a}}$} \\
\hline & Serous $(n=107)$ & Mucinous $(n=13)$ & Clear cell $(n=50)$ & Endometrioid $(n=33)$ & $\begin{array}{l}\text { Undifferentiated } \\
\qquad(n=23)\end{array}$ & \\
\hline $\operatorname{IFN} \gamma \mid$ & $18.2(0.6-403.2)$ & $1.4(0.6-225.1)$ & $3.1(0.2-268.3)$ & $1.4(0.3-288.3)$ & $12.7(0.6-96.0)$ & 0.001 \\
\hline TNF $\alpha$ level & $4.0(0.6-1600.0)$ & $4.0(0.6-242.0)$ & $4.0(0.6-632.0)$ & $6.2(0.6-137.0)$ & $4.0(0.6-13.1)$ & 0.41 \\
\hline IL6 level & $1440.0(1.1-15600.0)$ & $147.0(22.9-1870.0)$ & $1710.0(1.0-15800.0)$ & $425.0(0.5-13700.0)$ & $113.0(0.5-12000.0)$ & 0.10 \\
\hline IL4 level & $6.93(0.45-720.0)$ & $6.9(0.8-11.9)$ & $5.9(0.5-16.6)$ & $7.8(0.3-18.3)$ & $6.9(0.5-14.2)$ & 0.30 \\
\hline IL10 level & $6.9(0.6-129.0)$ & $3.9(0.6-8.1)$ & $5.1(0.6-105.0)$ & $5.3(0.6-83.3)$ & $4.2(0.6-29.9)$ & 0.03 \\
\hline IL17a level & $9.5(1.8-42.1)$ & $27.0(3.2-36.0)$ & $20.8(1.1-42.0)$ & $27.0(1.8-43.2)$ & $20.1(1.0-39.0)$ & $<0.001$ \\
\hline IL17f level & $19.8(13.0-1710.0)$ & $19.1(16.0-39.5)$ & $21.0(16.0-530.0)$ & $20.9(16.0-172.0)$ & $21.0(16.4-51.0)$ & 0.56 \\
\hline IL21 level & $135.0(19.9-963.0)$ & $65.0(25.2-679.1)$ & $125.8(11.4-1206.0)$ & $100.8(13.0-1154.0)$ & $112.2(32.0-736.0)$ & 0.10 \\
\hline IL22 level & $12.4(3.8-130.0)$ & $16.0(4.5-24.0)$ & $14.8(6.1-52.8)$ & $10.3(4.3-44.0)$ & $11.1(1.2-43.0)$ & 0.52 \\
\hline IL23 level & $16.0(8.0-770.0)$ & $18.0(11.2-23.0)$ & $18.0(10.8-67.0)$ & $18.0(10.0-76.0)$ & $16.0(10.0-28.3)$ & 0.004 \\
\hline
\end{tabular}

IFN $\gamma$, interferon gamma; TNF $\alpha$, tumor necrosis factor alpha; IL, interleukin.

${ }^{a}$ By Kruskal-Wallis test.

http://erc.endocrinology-journals.org DOI: 10.1530/ERC-15-0145
(C) 2015 Society for Endocrinology Printed in Great Britain
Published by Bioscientifica Ltd 
(A) Disease relapse

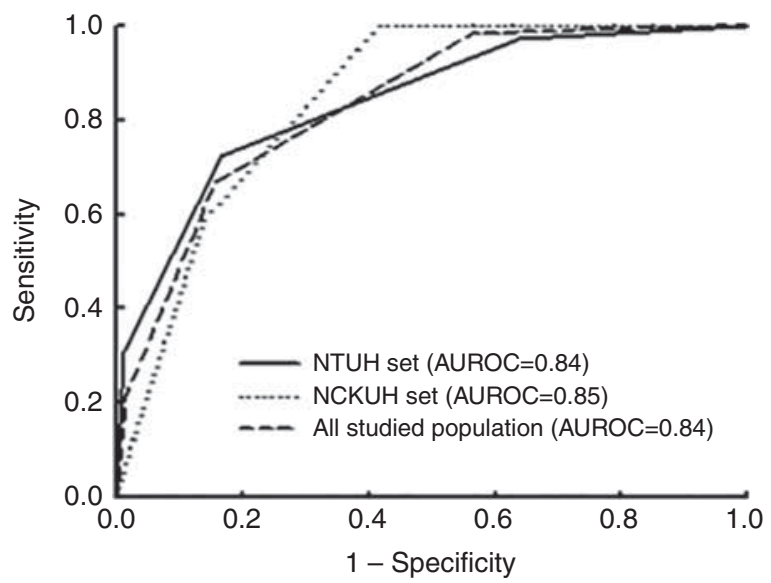

(B) Disease-related death

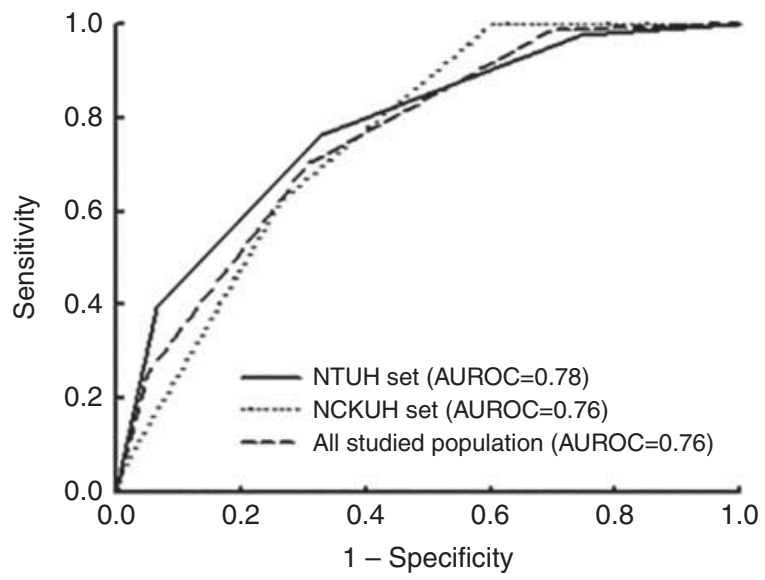

Figure 1

Receiver operating characteristic (ROC) curves of the overall score for disease relapse (A) and disease-related death (B) of ovarian cancer patients in the derivation group (NTUH set), validation group (NCKUH set), and all patients. NTUH, National Taiwan University Hospital; NCKUH, National Cheng Kung University Hospital; AUROC, area under the ROC curves.

course of cancer patients (Yigit et al. 2011, Cândido et al. 2013, Chen et al. 2013). Theoretically, a combination of clinical parameters and cytokine profiles in ascites could provide a predictive system to evaluate the outcomes of ovarian cancer patients. The present study demonstrated that a combination of surgical status, IL17a and IL21 expression levels in ascites could be used in a practical risk-scoring system to predict the outcome of patients (Table 5), and this system was validated by the derivation group (NTUH set), the validation group (NCKUH set) and all of the studied patients (Fig. 1). Furthermore, good correlations between the overall predictive risk scores and survival of the patients with ovarian cancer were noted by sub-grouping all of the patients into four groups (Fig. 2).

$\mathrm{CD} 4^{+} \mathrm{T}$ cells can differentiate into different lineages of Th cells with distinct developmental regulation and biological functions. Th17 cells have been identified as a new lineage of effector Th cells, and shown to be important in immune responses to various immune diseases, including malignancies. The Th17-related cytokines include IL17a, IL17f, IL21, IL22, and IL23 (Dong 2008). Fialová et al. suggested that the development of antitumor immune responses in ovarian cancer patients is highly dynamic. The recruitment of Th17 cells with higher IL17 and IL21 expression levels in ovarian cancer tissues has been reported in the early stages of disease (Fialová et al. 2013), and a lower level of IL17 has been reported in more advanced ovarian cancer-associated ascites (Kryczek et al. 2009). The expression levels of IL17a and IL21 in
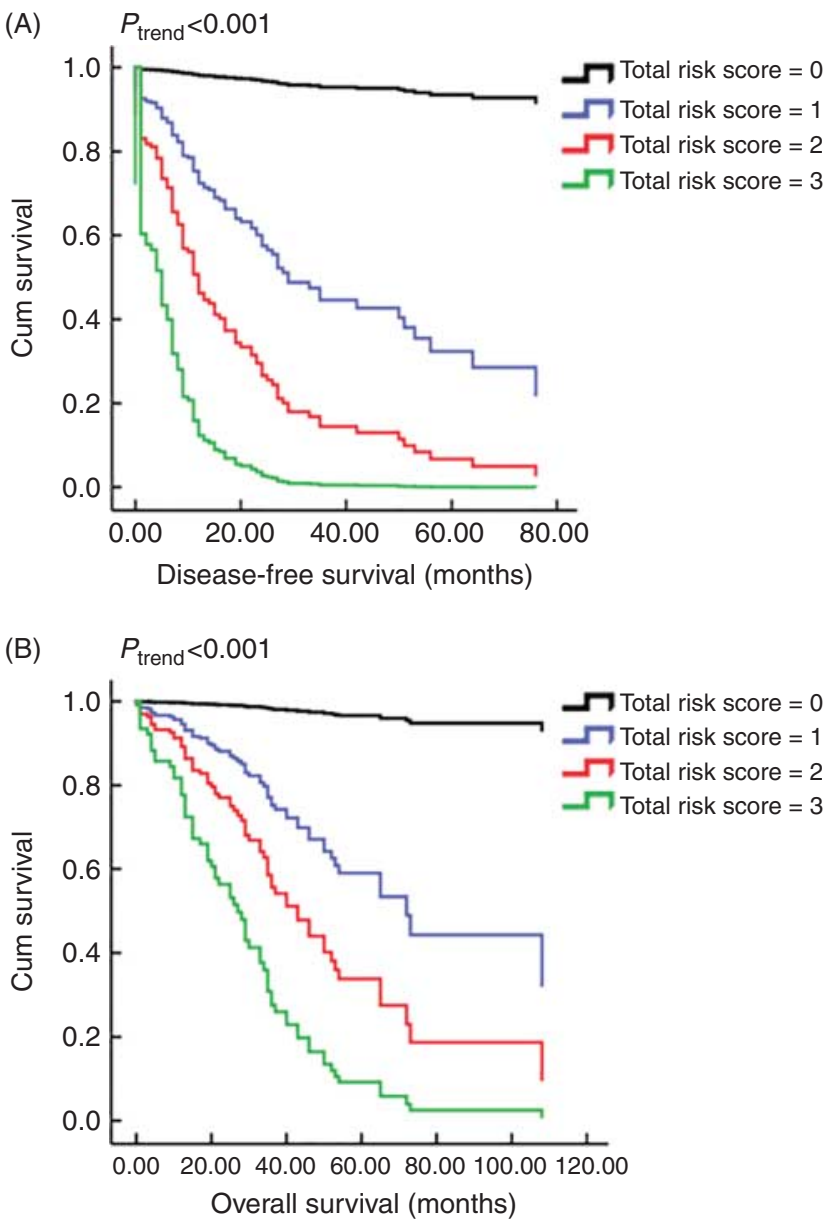

Figure 2

(A) Survival curves for disease-free survival of all 226 ovarian cancer patients based on the overall scores. (B) Overall survival of all 226 ovarian cancer patients based on the overall scores.

Published by Bioscientifica Ltd 
ascites were important predictors for the OS of ovarian cancer patients in this study (Table 5). Th17-related cytokines may therefore play a crucial role in the outcomes of ovarian cancer patients.

Presently, clinical trials focusing on Th17 and its related cytokines for cancer treatment are ongoing, especially for ovarian carcinoma. IL17 has been reported to potentially be a favorable prognostic marker of antitumor immune response and an indicator of the chemosensitivity of ovarian carcinoma (Kryczek et al. 2009, Droeser et al. 2013). Thus, targeting Th17 and its related cytokines may be a potential strategy for the treatment of ovarian cancer. After being validated by a prospective clinical trial with more participants, measuring the expression levels of Th17-related cytokines in ascites and then applying the risk-scoring system developed in this study may be helpful before providing current chemotherapeutic agents or cancer immunotherapy for ovarian cancer patients. In addition, alternative therapeutic methods would be considered and developed for cases with unfavorable expression profiles of Th17related cytokines in their ascites.

Alterations of immunocytes and cytokines in the tumor microenvironment have been reported to be related to the prognosis of ovarian cancer patients in several previous studies (Zhang et al. 2003, Yigit et al. 2011, Cândido et al. 2013, Chen et al. 2013, Webb et al. 2014). To the best of our knowledge, no current risk-scoring system includes immune-related factors. Therefore, the first significant strength of this study is that we developed and validated a practical risk-scoring system with IL17a, IL21 and surgical status to predict the survival of ovarian cancer patients. Second, our study showed that the Th17related cytokines, IL17a and IL21, may have the potential to be prognostic and therapeutic biomarkers.

There are several limitations to this study. Differences in the levels of Th17-related cytokines expressed in serum and ascites were not explored. In addition, the relationship between Th17-related cytokine levels and variations in immune effector and suppressor cells in ascites were not investigated either. Therefore, further studies are needed to evaluate the underlying immunological regulation in the ovarian cancer microenvironment.

We previously reported that IFN $\gamma$ levels in ascites could independently predict the OS of ovarian cancer patients (Chen et al. 2013). To explore more potential immunological predictors, Th1-, Th2-, and Th17-related cytokines were included in the current survey. In addition to IL17a and IL21, the expression level of IFN $\gamma$ in ascites still significantly influenced the OS of the 144 patients in univariate analysis (Table 4). However, only IL17a and IL21 showed significant impacts on the OS in multivariate analysis (Table 5). Therefore, IL17a and IL21 may play more important roles in carcinogenesis; however, further studies are needed to elucidate the associated mechanisms.

In conclusion, we used a combination of clinical and immunologic parameters to develop a practical scoring system to predict the outcome of patients with ovarian carcinoma. Good correlations between the overall predictive risk score and survival of the ovarian cancer patients were demonstrated. In addition, IL17a and IL21 may have the potential to be therapeutic biomarkers.

\section{Supplementary data}

This is linked to the online version of the paper at http://dx.doi.org/10.1530/ ERC-15-0145

\section{Declaration of interest}

The authors declare that there is no conflict of interest that could be perceived as prejudicing the impartiality of the research reported.

\section{Funding}

This work was supported in part by the 7th core laboratory facilities of the Department of Medical Research of NTUH and by grants from the Ministry of Science and Technology (MOST 102-2321-B-002-080-MY2 and 103-2314B-281-008-MY3), NTUH (UN103-092), and Cathay General Hospital-NTUH Joint Research Program (102-CGN06 and 103-CGN01).

\section{Acknowledgements}

The authors thank Drs San-Lin You and Mei-Huei Chen for statistical assistance.

\section{References}

Bast RC Jr, Hennessy B \& Mills GB 2009 The biology of ovarian cancer: new opportunities for translation. Nature Reviews. Cancer 9 415-428. (doi:10.1038/nrc2644)

Cândido EB, Silva LM, Carvalho AT, Lamaita RM, Filho RM, Cota BD \& da Silva-Filho AL 2013 Immune response evaluation through determination of type 1 , type 2 and type 17 patterns in patients with epithelial ovarian cancer. Reproductive Sciences 20 828-837. (doi:10.1177/ 1933719112466299)

Cannistra SA 2004 Cancer of the ovary. New England Journal of Medicine 351 2519-2529. (doi:10.1056/NEJMra041842)

Casey SC, Amedei A, Aquilano K, Azmi AS, Benencia F, Bhakta D, Bilsland AE, Boosani CS, Chen S, Ciriolo MR et al. 2015 Cancer prevention and therapy through the modulation of the tumor microenvironment. Seminars in Cancer Biology [in press]. (doi:10.1016/j.semcancer.2015.02.007)

Chan J, Java J, Monk B, Alvarez-Secord A, Kapp D, Birrer M, Aghajanian C, Bookman M, Kattan M \& Burger R 2012 A practical prediction model for determining bevacizumab response and toxicity in the treatment of

Published by Bioscientifica Ltd 
advanced ovarian and peritoneal cancers - an analysis of GOG 218. Gynecologic Oncology 125 S31. (doi:10.1016/j.ygyno.2011.12.070)

Chan PC, Shinn-Forng Peng S, Chiou MY, Ling DL, Chang LY, Wang KF, Fang CT \& Huang LM 2014 Risk for tuberculosis in child contacts. Development and validation of a predictive score. American Journal of Respiratory and Critical Care Medicine 189 203-213. (doi:10.1164/rccm. 201305-0863OC)

Chen YL, Chang MC, Chen CA, Lin HW, Cheng WF \& Chien CL 2012a Depletion of regulatory $\mathrm{T}$ lymphocytes reverses the imbalance between pro- and anti-tumor immunities via enhancing antigen-specific $\mathrm{T}$ cell immune responses. PLOS ONE 7 e47190. (doi:10.1371/journal.pone. 0047190)

Chen YL, Chang MC, Huang CY, Chiang YC, Lin HW, Chen CA, Hsieh CY $\&$ Cheng WF $2012 b$ Serous ovarian carcinoma patients with high $\alpha$-folate receptor had reducing survival and cytotoxic chemo-response. Molecular Oncology 6 360-369. (doi:10.1016/j.molonc.2011.11.010)

Chen YL, Cheng WF, Chang MC, Lin HW, Huang CT, Chien CL \& Chen CA 2013 Interferon- $\gamma$ in ascites could be a predictive biomarker of outcome in ovarian carcinoma. Gynecologic Oncology 131 63-68. (doi:10.1016/j. ygyno.2013.07.105)

Chi DS, Palayekar MJ, Sonoda Y, Abu-Rustum NR, Awtrey CS, Huh J, Eisenhauer EL, Barakat RR \& Kattan MW 2008 Nomogram for survival after primary surgery for bulky stage IIIC ovarian carcinoma. Gynecologic Oncology 108 191-194. (doi:10.1016/j.ygyno.2007.09.020)

Cooke SL, Ng CK, Melnyk N, Garcia MJ, Hardcastle T, Temple J, Langdon S, Huntsman D \& Brenton JD 2010 Genomic analysis of genetic heterogeneity and evolution in high-grade serous ovarian carcinoma. Oncogene 29 4905-4913. (doi:10.1038/onc.2010.245)

Curiel TJ, Coukos G, Zou L, Alvarez X, Cheng P, Mottram P, Evdemon-Hogan M, Conejo-Garcia JR, Zhang L, Burow M et al. 2004 Specific recruitment of regulatory $\mathrm{T}$ cells in ovarian carcinoma fosters immune privilege and predicts reduced survival. Nature Medicine $\mathbf{1 0}$ 942-949. (doi:10.1038/nm1093)

Dong C 2008 TH17 cells in development: an updated view of their molecular identity and genetic programming. Nature Reviews. Immunology 8 337-348. (doi:10.1038/nri2295)

Droeser RA, Güth U, Eppenberger-Castori S, Stadlmann S, Hirt C, Terracciano L \& Singer G 2013 High IL-17-positive tumor immune cell infiltration is indicative for chemosensitivity of ovarian carcinoma. Journal of Cancer Research and Clinical Oncology 139 1295-1302. (doi:10.1007/s00432-013-1441-1)

Fialová A, Partlová S, Sojka L, Hromádková H, Brtnický T, Fučíková J, Kocián P, Rob L, Bartůňková J \& Spíšek R 2013 Dynamics of T-cell infiltration during the course of ovarian cancer: the gradual shift from a Th17 effector cell response to a predominant infiltration by regulatory T-cells. International Journal of Cancer 132 1070-1079. (doi:10.1002/ ijc.27759)

Fridman WH, Pagès F, Sautès-Fridman C \& Galon J 2012 The immune contexture in human tumours: impact on clinical outcome. Nature Reviews. Cancer 12 298-306. (doi:10.1038/nrc3245)

Hanahan D \& Weinberg RA 2011 Hallmarks of cancer: the next generation. Cell 144 646-674. (doi:10.1016/j.cell.2011.02.013)

Hirahara N, Nio Y, Sasaki S, Minari Y, Takamura M, Iguchi C, Dong M, Yamasawa K \& Tamura K 2001 Inoculation of human interleukin-17 genetransfected Meth-A fibrosarcoma cells induces T cell-dependent tumorspecific immunity in mice. Oncology 61 79-89. (doi:10.1159/000055357)

International Federation of Gynecology and Obstetrics 1971 Classification and staging of malignant tumours in the female pelvis. Acta Obstetricia et Gynecologica Scandinavica 50 1-7. (doi:10.3109/00016347109157278)
Jemal A, Bray F, Center MM, Ferlay J, Ward E \& Forman D 2011 Global cancer statistics. CA: A Cancer Journal for Clinicians 61 69-90. (doi:10.3322/caac.20107)

Kim A, Ueda Y, Naka T \& Enomoto T 2012 Therapeutic strategies in epithelial ovarian cancer. Journal of Experimental \& Clinical Cancer Research 31 14. (doi:10.1186/1756-9966-31-14)

Kryczek I, Banerjee M, Cheng P, Vatan L, Szeliga W, Wei S, Huang E, Finlayson E, Simeone D, Welling TH et al. 2009 Phenotype, distribution, generation, and functional and clinical relevance of Th17 cells in the human tumor environments. Blood 114 1141-1149. (doi:10.1182/blood-2009-03-208249)

Kurman RJ \& Shih IeM 2010 The origin and pathogenesis of epithelial ovarian cancer: a proposed unifying theory. American Journal of Surgical Pathology 34 433-443. (doi:10.1097/PAS. Ob013e3181cf3d79)

Latha TS, Panati K, Gowd DS, Reddy MC \& Lomada D 2014 Ovarian cancer biology and immunotherapy. International Reviews of Immunology 33 428-440. (doi:10.3109/08830185.2014.921161)

Oreja-Guevara C, Ramos-Cejudo J, Aroeira LS, Chamorro B \& Diez-Tejedor E 2012 TH1/TH2 cytokine profile in relapsing-remitting multiple sclerosis patients treated with Glatiramer acetate or Natalizumab. BMC Neurology 12 95. (doi:10.1186/1471-2377-12-95)

Previs RA, Bevis KS, Huh W, Tillmanns T, Perry L, Moore K, Chapman J, McClung C, Kiet T, Java J et al. 2014 A prognostic nomogram to predict overall survival in women with recurrent ovarian cancer treated with bevacizumab and chemotherapy. Gynecologic Oncology 132 531-536. (doi:10.1016/j.ygyno.2014.01.036)

Raspollini MR, Castiglione F, Rossi Degl'innocenti D, Amunni G, Villanucci A, Garbini F, Baroni G \& Taddei GL 2005 Tumour-infiltrating $\gamma / \delta$ T-lymphocytes are correlated with a brief disease-free interval in advanced ovarian serous carcinoma. Annals of Oncology 16 590-596. (doi:10.1093/annonc/mdi112)

Suh KS, Park SW, Castro A, Patel H, Blake P, Liang M \& Goy A 2010 Ovarian cancer biomarkers for molecular biosensors and translational medicine. Expert Review of Molecular Diagnostics 10 1069-1083. (doi:10.1586/erm.10.87)

Tomsová M, Melichar B, Sedláková I \& Steiner I 2008 Prognostic significance of $\mathrm{CD}^{+}$tumor-infiltrating lymphocytes in ovarian carcinoma. Gynecologic Oncology 108 415-420. (doi:10.1016/j.ygyno. 2007.10.016)

Vaughan S, Coward JI, Bast RC Jr, Berchuck A, Berek JS, Brenton JD, Coukos G, Crum CC, Drapkin R, Etemadmoghadam D et al. 2011 Rethinking ovarian cancer: recommendations for improving outcomes. Nature Reviews. Cancer 11 719-725. (doi:10.1038/nrc3144)

Wang DH, Guo L \& Wu XH 2015 Checkpoint inhibitors in immunotherapy of ovarian cancer. Tumour Biology 36 33-39. (doi:10.1007/s13277014-2848-2)

Webb JR, Milne K, Watson P, Deleeuw RJ \& Nelson BH 2014 Tumorinfiltrating lymphocytes expressing the tissue resident memory marker CD103 are associated with increased survival in high-grade serous ovarian cancer. Clinical Cancer Research 20 434-444. (doi:10.1158/ 1078-0432.CCR-13-1877)

Yigit R, Figdor CG, Zusterzeel PL, Pots JM, Torensma R \& Massuger LF 2011 Cytokine analysis as a tool to understand tumour-host interaction in ovarian cancer. European Journal of Cancer 47 1883-1889. (doi:10.1016/ j.ejca.2011.03.026)

Zhang L, Conejo-Garcia JR, Katsaros D, Gimotty PA, Massobrio M, Regnani G, Makrigiannakis A, Gray H, Schlienger K, Liebman MN et al. 2003 Intra-tumoral $\mathrm{T}$ cells, recurrence, and survival in epithelial ovarian cancer. New England Journal of Medicine 348 203-213. (doi:10.1056/ NEJMoa020177)

Received in final form 18 June 2015

Accepted 6 July 2015

Made available online as an Accepted Preprint

6 July 2015

Published by Bioscientifica Ltd http://erc.endocrinology-journals.org

DOI: 10.1530/ERC-15-0145
(C) 2015 Society for Endocrinology Printed in Great Britain 